\title{
Florística e estrutura do estrato arbóreo de uma floresta de várzea no município de Santa Bárbara do Pará, Estado do Pará, Brasil
}

\author{
Gideão Costa dos SANTOS', Mário Augusto Gonçalves JARDIM²
}

\section{RESUMO}

Este estudo teve como objetivo conhecer a composição florística e a estrutura do estrato arbóreo de uma floresta de várzea na localidade de São José do Aracy, no município de Santa Bárbara do Pará, estado do Pará, Brasil. Foram instaladas quatro parcelas de 100 x $100 \mathrm{~m}$ subdivididas em cinco transectos de 20 × $100 \mathrm{~m}$ e foram amostradas as espécies arbóreas lenhosas e palmeiras. Para as espécies lenhosas adotou-se diâmetro à altura do peito (DAP) ${ }^{3} 10 \mathrm{~cm}$ e para palmeiras a circunferência à altura do peito $(\mathrm{CAP})^{3}$ $10 \mathrm{~cm}$ e estimou-se a altura. O material botânico coletado foi identificado em nível de família, gênero e espécie, sendo incorporado ao herbário João Murça Pires do Museu Paraense Emílio Goeldi (MPEG). Na análise fitossociológica foram calculados os parâmetros de Densidade Relativa (DR\%), Freqüência Relativa (FR\%), Dominância Relativa (DOMR\%), Índice de Valor de Importância (IVI), Índice de Valor de Cobertura (IVC), Índice de similaridade de Sorenson (S'), Índice de diversidade de Shannon (H') e Equabilidade (E’). Os resultados mostraram 29 famílias, 58 gêneros e 70 espécies. As famílias com maior diversidade foram Caesalpiniaceae, Mimosaceae e Papilionaceae, com nove, oito e sete espécies, respectivamente; Arecaceae e Papilionaceae obtiveram maior densidade (27,75\% e 25,84\%); treze famílias foram mais freqüentes com 4,88\% entre elas; Papilionaceae com maior dominância e índice de valor de importância (37,50\% e 68,21); Euterpe oleracea Mart. apresentou maior densidade relativa e Pterocarpus officinalis Jacq., maior dominância relativa, maior índice de valor de importância e de cobertura. $\mathrm{O}$ índice de diversidade de 2,63 foi considerado baixo, com equabilidade de 0,63 e similaridade de 0,60. A várzea estudada apresentou baixa diversidade em espécies.

\section{PALAVRAS-CHAVE}

Açaizeiro, várzea, composição florística, estrutura, diversidade.

\section{Floristic and structure of tree communities in the floodplain forest on municipallity of Santa Bárbara do Pará, State of Pará, Brazil}

\section{ABSTRACT}

This research has taken place in four of $100 \times 100 \mathrm{~m}$ in São José do Aracy, in the municipallity of Santa Bárbara do Pará, State of Pará, Brazil. Each part was divided in five transects of $20 \times 10 \mathrm{~m}$. The arboreal woody species with $\mathrm{DBH}^{3} 10 \mathrm{~cm}$ and palms with $\mathrm{CBH} 10 \mathrm{~cm}$ were evaluated and the height was estimated. This collected botanical material was identified in family, genera and species, and was incorporated in the João Murça Pires Herbarium, at Museu Paraense Emilio Goeldi (MPEG). For phytosociology analysis were calculated Relative Density (DR), Relative Frequency (FR), Relative Dominance (DOMR), Index Value of Importance (IVI), Index of Value of Coberture (IVC) and this Index of similarity of Sorenson (S'), Index of Diversity of Shannon (H') and Equability (E'). These results showed 29 families, 58 genera and 70 species. These families with major diversity were Caesalpiniaceae, Mimosaceae and Papilionaceae, with nine, eight and seven species respectively. Arecaceae and Papilionaceae obtained major density (27.75\% and 25.84\%); thirteen families were frequently with 4.88\%; Papilionaceae obtained major dominance and Index value of importance (37.50\% and 68.21\%); Euterpe oleracea Mart. presented major relative density while Pterocarpus officinalis Jacq. presented major relative dominance, major Index of value of importance and coberture. This Index of diversity of 2.63 was considered low, with equability of 0.63 and similarity of 0.60 . The floodplain forest presented low diversity in the species.

\section{KEY WORDS}

Açai palm, floodplain, floristic composition, structure, diversity.

\footnotetext{
1, 2 Museu Paraense Emílio Goeldi. Coordenação de Botânica. Av. Tancredo Neves, n.1901, caixa postal 399. CEP 66040-170. Belém - PA; (gcsantos@museu-goeldi.br; jardim@museu-goeldi.br)
} 


\section{INTRODUÇÃO}

É comum nas várzeas a presença de determinadas famílias e espécies, pois são áreas com condiçôes restritas, ou seja, solos com alto nível de saturação, fluxo constante de maré, período e altura de inundação, salinidade etc (Jardim et al 2004). No entanto, certas espécies tendem a ser mais abundantes como p.ex. Protium sagotianum Marchand (breu-branco); Protium sp.(breu-preto); Caryocar microcarpum Ducke (piquiarana); Cassia pentandra Raddi (fava-marimari); Dipteryx odorata (Aubl.) Willd. (cumaru); Symphonia globulifera L.f. (anani); Couratari courbaril Aubl. (tauari) e Carapa guianensis Aubl. (andiroba), constatadas no estudo de Martorano (2000) em 1,95 ha de floresta de várzea na bacia do Una, em Belém e Euterpe oleracea Mart. (açaí), citada nos trabalhos de composição florística em floresta de várzea com relevante índice de valor de importância (Jardim \& Cunha 1998). Sendo assim, é perceptível uma estreita relação entre esses grupos botânicos e o meio ambiente.

Nos estudos florísticos em floresta de várzea citam-se Macedo \& Anderson (1993) sobre a densidade e área basal de Virola surinamensis (Rol. ex Rottb.) Warb. e ocorrência de Leguminosae e Guttiferae em um hectare de floresta de várzea no município de Breves (PA) e Pires \& Koury (1959); Pires (1973); Ayres (1993) e Almeida et al. (2002) que consideraram essas áreas com pouca diversidade florística, no entanto, com espécies de importância econômica. $\mathrm{Na}$ estrutura das populaçōes, diversas famílias e espécies são dominantes, como exemplo, Conceição (1990), em uma floresta de várzea no município de Colares PA, mostrou que Meliaceae foi representada por Carapa guianensis Aubl. e Arecaceae por Euterpe oleracea Mart. Desta forma, Rabelo (1999), em estudo realizado nas áreas estuarinas do Estado do Amapá, ressaltou que espécies, quando comparadas em diferentes várzeas, podem apresentar padrōes diferentes de distribuição, o que pode estar relacionado a diferentes fatores bióticos do meio, ao índice pluviométrico e ao movimento das marés, o que influenciará na regeneração natural.

Diversos fatores podem ser determinantes ou não no estabelecimento das espécies nas florestas de várzeas. Um deles refere-se à topografia, onde grupos desenvolvem-se em áreas mais altas e outros em áreas mais baixas, pois, segundo Lima et al. (2000), na elevação dos solos de várzea há predominância de espécies arbóreas dicotiledôneas como Hevea brasiliensis (Willd. ex A. Juss.) Mull.Arg. (seringueira), Virola surinamensis (Rol. ex Rottb.) Warb. (ucuúba), Ceiba pentandra (L.) Gaertn. (sumaúma) e Spondias mombin L. (taperebá). Na composiçāo florística, estrutura e, conseqüentemente, na fisionomia das várzeas, as palmeiras estão sempre presentes, fato este comprovado por Bonaide \& Bacon (1999), em Trinidad, onde Mauritia flexuosa L.f.e Roystonea oleracea (Jacq.) O.F.Cook são responsáveis pela maior abundância, freqüência, dominância e valor de importância. Evidenciam, ainda, as espécies arbóreas Manilkara bidentata (A.DC.) A. Chev, Carapa guianensis Aubl. e Sterculia pruriens (Aubl.) K. Schum., todas com valores estruturais inferiores ao das palmeiras. Outros estudos, como o de Sanchez et al. (1999), destacaram Arecaceae com quatro espécies e um elevado número de indivíduos.

As várzeas de Santa Bárbara do Pará, pelo advento da extração do palmito, sofreram intensa intervenção antrópica, resultando (informaçôes de moradores locais) na modificação da fisionomia da floresta. Neste sentido, esse estudo objetivou conhecer a composição e a estrutura florística nas várzeas do município de Santa Bárbara, estado do Pará.

\section{MATERIAL E MÉTODOS}

O município de Santa Bárbara do Pará está situado na mesorregião metropolitana de Belém sob as coordenadas geográficas $01^{\circ} 13^{\prime} 26^{\prime \prime}$ de latitude Sul e $48^{\circ} 17^{\prime} 22^{\prime \prime}$ de longitude Oeste, às margens da Rodovia Augusto Meira Filho PA-391, distando de Belém cerca de 40 km, (Fig. 1). Está dividido em dez localidades, dentre elas, São José do Aracy.

A localidade de São José do Aracy dista da sede do município $6,5 \mathrm{~km}$ e possui duas vias de acesso: o rio Aracy e a estrada do Aracy. Apresenta clima megatérmico úmido com temperaturas elevadas durante o ano todo, sendo a média em torno de $26^{\circ} \mathrm{Ce}$ os meses mais quentes, de setembro a dezembro. $\mathrm{O}$ índice pluviométrico chega a ultrapassar os $2.550 \mathrm{~mm}$ anuais, com a maior incidência no período de janeiro a junho. A umidade relativa do ar está em torno de $85 \%$. O solo é do tipo Glei Pouco Húmico Hidromórfico. A topografia caracteriza-se por um relevo predominantemente plano, fazendo parte do planalto rebaixado da Amazônia. No entorno, a vegetação é composta por floresta secundária e, nas áreas de várzea, por vegetação arbórea, cipós e açaizais, banhada pelo fluxo hidrológico de furos, rios e igarapés (Sudam, 1993).

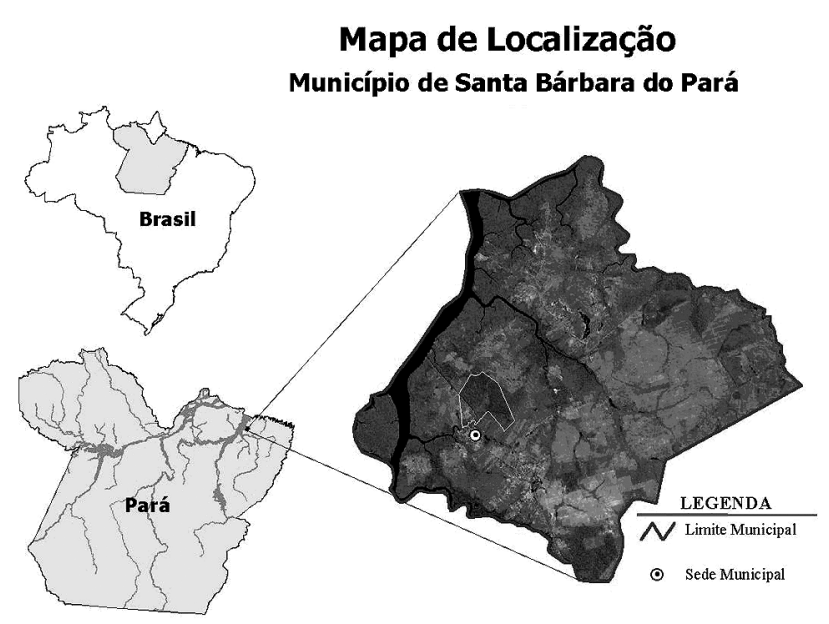

Figura 1 - Localização do município de Santa Bárbara do Pará, Estado do Pará, Brasil. Fonte: ALMEIDA et al. (2003). Adaptação: Altenir Pereira Sarmento, CBO/MPEG. 
Para coleta dos dados, foram utilizadas quatro parcelas de um hectare distribuídas aleatoriamente em pontos diferentes com as seguintes coordenadas geográficas: parcela $1\left(1^{\circ} 09^{\prime} 45^{\prime \prime}\right.$ de latitude Sul e $48^{\circ} 17^{\prime} 18^{\prime \prime}$ de longitude Oeste); parcela $2\left(1^{\circ} 09^{\prime}\right.$ $50^{\prime \prime}$ de latitude Sul e $48^{\circ} 17^{\prime} 04^{\prime \prime}$ de longitude Oeste); parcela 3 ( ${ }^{\circ} 09^{\prime} 24^{\prime \prime}$ de latitude Sul e $48^{\circ} 17^{\prime} 30^{\prime \prime}$ de longitude Oeste) e parcela $4\left(1^{\circ} 09^{\prime} 42^{\prime \prime}\right.$ de latitude Sul e $48^{\circ} 17^{\prime} 22^{\prime \prime}$ de longitude Oeste). Cada parcela foi subdividida em cinco transectos de 20 x $100 \mathrm{~m}$, onde foi realizado o levantamento florístico das espécies arbóreas lenhosas e das palmeiras. Para as espécies arbóreas lenhosas, foram adotadas mediçôes de diâmetro à altura do peito - DAP ${ }^{3} 10 \mathrm{~cm}$ e para os açaizais, circunferência à altura do peito - CAP ${ }^{3} 10 \mathrm{~cm}$. O material botânico foi coletado e identificado no herbário do Museu Paraense Emílio Goeldi - MPEG em nível de família, gênero e espécie, utilizando-se o sistema de Cronquist (1981).

Os dados das espécies arbóreas lenhosas foram convertidos de DAP para CAP para evitar discrepâncias de valores entre espécies lenhosas e palmeiras, especialmente em relação ao açaizeiro e, com auxílio dos programas Excell e Fitopac (desenvolvido pelo Prof. Dr. John Shepherd, do Instituto de Biologia da UNICAMP-SP), foi calculada a Densidade Relativa (DR), Freqüência Relativa (FR), Dominância Relativa (DMOR), Índice de Valor de Importância (IVI) e Índice de Valor de Cobertura (IVC). Na estratificação dos indivíduos em classes diamétricas, os valores das circunferências foram convertidos para DAP de modo a minimizar o número de classes que foram: I (3-10 unidade); II (10,1-20); III (20,1-30); IV (30,1-40) e V (>40). Para a altura, estabeleceram-se as seguintes classes: I (1-5 m); II (5,1-10 m); III (10,1-15 m); IV (15,1-20 m); V (20,1-25 m) e VI $(>25 \mathrm{~m})$.

Demais parâmetros utilizados na análise florística como Índice de Similaridade Florística foi analisado pelo método de Sorenson $[S=2 c /(a+b)]$ onde, $a=$ número de espécies da parcela $a ; b=$ número de espécies da parcela $\mathrm{b} ; \mathrm{c}=$ número de espécies comuns às parcelas a e b, (Legendre \& Legendre,1983); Índice de Diversidade calculado pelo método de Shannon-Weaver $\left(\mathrm{H}^{\prime}=\right.$ Ópi x Ln x pi) Magurran (1988) e Equabilidade (E'), (E'= H'/ Ln $x$ S) onde: $\mathrm{H}^{\prime}=$ índice de Shannon; $S=$ número de espécies, (Pielou, 1977).

\section{RESULTADOS}

Em geral, as várzeas do município de Santa Bárbara do Pará apresentaram vegetação arbórea composta por espécies lenhosas e palmeiras, cipós e dossel não contínuo. O levantamento florístico resultou num total de 3.286 indivíduos distribuídos em 29 famílias, 58 gêneros e 70 espécies. Das famílias encontradas, as mais bem representadas em número de espécies foram: Caesalpiniaceae (nove espécies), Mimosaceae (oito espécies), Papilionaceae (sete espécies) distribuídas em dois grandes grupos: Magnoliopsida (28 famílias) e Liliopsida (uma família), representada por Arecaceae. As famílias e os parâmetros fitossociológicos encontrados nos quatro hectares estão listados na Tab. 1 por ordem de Índice de Valor de Importância.

Arecaceae, Papilionaceae e Caesalpiniaceae apresentaram maiores valores de densidade relativa, sendo $27,75 \% ; 25,84 \% \mathrm{e}$ $16,34 \%$, respectivamente. $\mathrm{Na}$ freqüência relativa houve similaridade entre treze famílias com valor de $4,88 \%$, entre elas, as de maior densidade, correspondendo à ocorrência nas quatro parcelas. Papilionaceae (37,50\%), Caesalpiniaceae $(13,67 \%)$ e Clusiaceae $(8,24 \%)$ foram as de maior dominância. Desse modo, o maior IVI (índice de valor de importância) na comunidade foi o de Papilionaceae $(68,21)$ seguido por Arecaceae $(38,89)$ e Caesalpiniaceae $(34,89)$.

A análise geral das espécies nas quatro parcelas encontra-se listada por índice de valor de importância na Tab. 2. Em relaçãoo às espécies com maiores números de indivíduos, destacaram-se Euterpe oleracea (açaí) com 857 indivíduos e Pterocarpus officinalis (mututi) com 702, Macrolobium angustifolium (ingarana) e Pentaclethra macroloba (pracaxi) com 244 e 223. E. oleracea e P. officinalis apresentaram maior densidade relativa $26,08 \%$ e $21,36 \%$, seguida por $M$. angustifolium 7,43\% e $P$. macroloba, $6,79 \%$. Na freqüência relativa, 15 espécies apresentaram valor de 2,55\%, correspondendo à ocorrência em todas as quatro parcelas analisadas, dentre elas, as espécies com maior densidade citadas anteriormente. Na dominância relativa destacou-se Pterocarpus officinalis com 32,66\%, seguida de Symphonia globulifera e Avicennia germinans foram menos expressivas, porém, sobressaíram-se das demais espécies com 7,30\% e 6,76\%. As espécies com maior índice de valor de importância (IVI) foram $P$. officinalis, 56,57 e Euterpe oleracea, 32,62 com índice de valor de cobertura (IVC) de 54,03 e 30,08.

A área basal resultou a média de $26,29 \mathrm{~m}^{2} \cdot \mathrm{ha}^{-1}$ para os 3.286 indivíduos. Papilionaceae obteve o valor de 39,44 $\mathrm{m}^{2} \cdot \mathrm{ha}^{-1}$, mais que o dobro em relação à segunda Caesalpiniaceae $14,38 \mathrm{~m}^{2}$.ha ${ }^{1}$ e em relação à Arecaceae foi, de aproximadamente, o sêxtuplo de seu valor, $6,58 \mathrm{~m}^{2} \cdot \mathrm{ha}^{-1}$ (Fig.2). As espécies mais representativas em área basal, como mostra a Fig. 3 foram: $P$. officinalis com $34,36 \mathrm{~m}^{2}$.ha $\mathrm{a}^{-1}$, bem acima das demais espécies. Em comparaçāo à quinta colocada Rhizophora mangle L., P. officinalis foi maior seis vezes. E. oleracea, que é uma espécie de alta densidade, apresentou a sétima maior área basal: $4,20 \mathrm{~m}^{2} \cdot \mathrm{ha}^{-1}$.

$\mathrm{Na}$ distribuição dos indivíduos em classes diamétricas, conforme observado na Fig. 4, houve alta concentração de espécimes nas classes iniciais I, II e III, destacando-se a classe II (10,1 - 20 unidade) com 1.482 indivíduos. As classes IV e V agruparam menor número de indivíduos, demonstrando que, à medida que aumenta o número de classes, menor éo número de representantes (distribuição "J" invertido). Na classe II, com maior concentração de indivíduos, a população de E. oleracea contribuiu com 622 indivíduos, o equivalente a $41,9 \%$ do total de representantes. 
Tabela 1 - Famílias, Número de espécies (Nosp); Número de indivíduos (Ni), Densidade Relativa (DR); Freqüência Relativa (FR); Dominância Relativa (DOMR) e Índice de Valor de Importância (IVI) encontrados em quatro hectares de floresta de várzea em Santa Bárbara do Pará, estado do Pará, Brasil.

\begin{tabular}{|c|c|c|c|c|c|c|}
\hline Famílias & $N^{\circ} s p$ & $\mathrm{Ni}$ & DR (\%) & FR (\%) & DOMR (\%) & IVI \\
\hline Papilionaceae & 7 & 849 & 25,84 & 4,88 & 37,50 & 68,21 \\
\hline Arecaceae & 3 & 912 & 27,75 & 4,88 & 6,26 & 38,89 \\
\hline Caesalpiniaceae & 9 & 537 & 16,34 & 4,88 & 13,67 & 34,89 \\
\hline Clusiaceae & 4 & 187 & 5,69 & 4,88 & 8,24 & 18,81 \\
\hline Meliaceae & 1 & 136 & 4,14 & 4,88 & 3,97 & 12,99 \\
\hline Verbenaceae & 1 & 30 & 0,91 & 4,88 & 6,76 & 12,55 \\
\hline Myristicaceae & 1 & 97 & 2,95 & 4,88 & 3,76 & 11,59 \\
\hline Mimosaceae & 8 & 117 & 3,56 & 4,88 & 2,80 & 11,24 \\
\hline Euphorbiaceae & 3 & 73 & 2,22 & 4,88 & 3,73 & 10,83 \\
\hline Bombacaceae & 3 & 102 & 3,10 & 4,88 & 2,49 & 10,47 \\
\hline Sterculiaceae & 2 & 21 & 0,64 & 4,88 & 0,50 & 6,02 \\
\hline Burseraceae & 2 & 16 & 0,49 & 3,66 & 0,47 & 4,61 \\
\hline Polygonaceae & 1 & 10 & 0,30 & 3,66 & 0,33 & 4,29 \\
\hline Chrysobalanaceae & 4 & 10 & 0,30 & 3,66 & 0,24 & 4,21 \\
\hline Caryocaraceae & 1 & 10 & 0,30 & 3,66 & 0,15 & 4,11 \\
\hline Myrtaceae & 2 & 8 & 0,24 & 3,66 & 0,20 & 4,10 \\
\hline Sapotaceae & 3 & 11 & 0,33 & 2,44 & 0,93 & 3,70 \\
\hline Ebenaceae & 1 & 6 & 0,18 & 2,44 & 0,16 & 2,78 \\
\hline Combretaceae & 1 & 3 & 0,09 & 2,44 & 0,13 & 2,66 \\
\hline Melastomataceae & 1 & 2 & 0,06 & 2,44 & 0,03 & 2,53 \\
\hline Malpighiaceae & 1 & 2 & 0,06 & 1,22 & 0,50 & 1,78 \\
\hline Icacinaceae & 1 & 1 & 0,03 & 1,22 & 0,08 & 1,33 \\
\hline Apocynaceae & 1 & 2 & 0,06 & 1,22 & 0,03 & 1,31 \\
\hline Aquifoliaceae & 1 & 1 & 0,03 & 1,22 & 0,02 & 1,27 \\
\hline Lauraceae & 1 & 1 & 0,03 & 1,22 & 0,02 & 1,27 \\
\hline
\end{tabular}

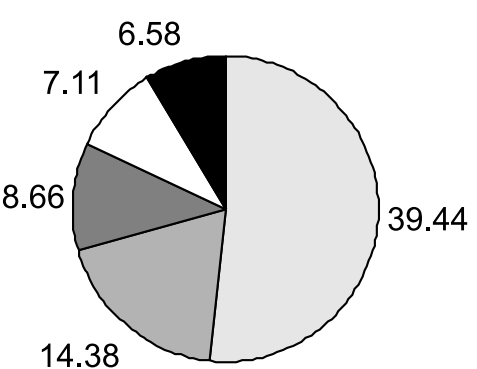

Figura 2 - Principais famílias com seus respectivos valores de área basal em 4 ha de floresta de várzea, em Santa Bárbara do Pará, estado do Pará, Brasil. $\square$ Papilionaceae

$\square$ Caesalpiniaceae

$\square$ Clusiaceae

$\square$ Verbenaceae

- Arecaceae

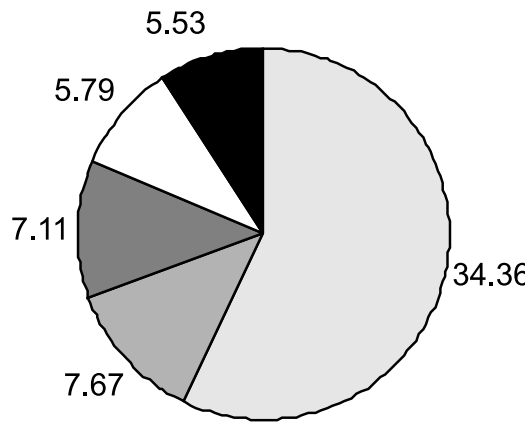

$\square$ Pterocarpus officinalis

$\square$ Symphonia globulifera

$\square$ Avicennia germinans $\square$ Macrolobium angustifolium Rhizophora mangle

Figura 3 - Espécies com maiores valores de área basal em 4 ha de floresta de várzea, em Santa Bárbara do Pará, estado do Pará. Brasil. 
Tabela 2 - Famílias, Espécies, Número de indivíduos (Ni); Densidade Relativa (DR); Freqüência Relativa (FR); Dominância Relativa (DOMR); Índice de Valor de Importância (IVI) e Índice de Valor de Cobertura (IVC) em 4ha de floresta de várzea em Santa Bárbara do Pará, estado do Pará, Brasil.

\begin{tabular}{|c|c|c|c|c|c|c|c|}
\hline Familias & Espécies & $\mathrm{Ni}$ & DR & FR & DOMR & IVI & IVC \\
\hline Papilionaceae & Pterocarpus officinalis Jacq. & 702 & 21,36 & 2,55 & 32,66 & 56,57 & 54,03 \\
\hline Arecaceae & Euterpe oleracea Mart. & 857 & 26,08 & 2,55 & 4,00 & 32,62 & 30,08 \\
\hline Caesalpiniaceae & Macrolobium angustifolium (Benth.) R.S.Cowan & 244 & 7,43 & 2,55 & 5,51 & 15,48 & 12,94 \\
\hline Clusiaceae & Symphonia globulifera L.f. & 142 & 4,32 & 2,55 & 7,30 & 14,16 & 11,62 \\
\hline Mimosaceae & Pentaclethra macroloba (Willd.) Kuntze & 223 & 6,79 & 2,55 & 4,70 & 14,04 & 11,49 \\
\hline Meliaceae & Carapa guianensis Aubl. & 136 & 4,14 & 2,55 & 3,97 & 10,66 & 8,11 \\
\hline Rhyzophoraceae & Rhizophora mangle L. & 82 & 2,50 & 1,91 & 5,26 & 10,30 & 7,76 \\
\hline Verbenaceae & Avicennia germinans (L.) L. & 30 & 0,91 & 2,55 & 6,76 & 10,22 & 7,67 \\
\hline Myristicaceae & Virola surinamensis (Rol. ex Rottb.) Warb. & 97 & 2,95 & 2,55 & 3,76 & 9,26 & 6,71 \\
\hline Caesalpiniaceae & Swartzia racemosa Benth. & 98 & 2,98 & 2,55 & 3,58 & 9,11 & 6,56 \\
\hline Euphorbiaceae & Hevea brasiliensis (Willd ex A. Juss.) M. Arg. & 68 & 2,07 & 2,55 & 3,66 & 8,27 & 5,73 \\
\hline Bombacaceae & Pachira aquatica Aubl. & 96 & 2,92 & 2,55 & 2,38 & 7,85 & 5,30 \\
\hline Papilionaceae & Platymiscium trinitatis Benth. & 47 & 1,43 & 2,55 & 2,07 & 6,05 & 3,50 \\
\hline Arecaceae & Attalea maripa (Aubl.) Mart. & 46 & 1,40 & 1,91 & 2,12 & 5,43 & 3,52 \\
\hline Lecythidaceae & Gustavia augusta L. & 39 & 1,19 & 2,55 & 1,12 & 4,85 & 2,30 \\
\hline Papilionaceae & Ormosia coutinhoi Ducke & 33 & 1,00 & 2,55 & 0,99 & 4,54 & 2,00 \\
\hline Papilionaceae & Vatairea guianensis Aubl. & 32 & 0,97 & 1,91 & 0,80 & 3,68 & 1,77 \\
\hline Sterculiaceae & Sterculia speciosa K. Schum. & 15 & 0,46 & 2,55 & 0,31 & 3,32 & 0,77 \\
\hline Mimosaceae & Inga nobilis Willd. & 22 & 0,67 & 1,91 & 0,58 & 3,6 & 1,25 \\
\hline Papilionaceae & Diplotropis martiusii Benth. & 25 & 0,76 & 1,91 & 0,34 & 3,01 & 1,10 \\
\hline Clusiaceae & Caraipa grandifolia Mart. & 19 & 0,58 & 1,91 & 0,47 & 2,96 & 1,04 \\
\hline Clusiaceae & Caraipa richardiana Cambess. & 18 & 0,55 & 1,91 & 0,33 & 2,79 & 0,88 \\
\hline Lecythidaceae & Eschweilera coriacea (DC.) S.A. Mori & 15 & 0,46 & 1,91 & 0,41 & 2,77 & 0,86 \\
\hline Polygonaceae & Coccoloba latifolia Lam. & 10 & 0,30 & 1,91 & 0,33 & 2,54 & 0,63 \\
\hline Caryocaraceae & Caryocar microcarpum Ducke & 10 & 0,21 & 1,91 & 0,15 & 2,36 & 0,45 \\
\hline Sapotaceae & Pouteria cuspidata (A. DC.) Baehni & 7 & 0,30 & 1,27 & 0,86 & 2,35 & 1,07 \\
\hline Myrtaceae & Eugenia belemitana McVaugh. & 7 & 0,21 & 1,91 & 0,18 & 2,31 & 0,40 \\
\hline Mimosaceae & Inga thibaudiana DC. & 7 & 0,21 & 1,91 & 0,14 & 2,27 & 0,35 \\
\hline Mimosaceae & Pithecellobium inaequale (Humb \& Bonpl. ex Willd.) Benth. & 14 & 0,43 & 1,27 & 0,53 & 2,23 & 0,95 \\
\hline Papilionaceae & Dipteryx odorata (Aubl.) Willd. & 9 & 0,27 & 1,27 & 0,62 & 2,17 & 0,89 \\
\hline Burseraceae & Protium heptaphyllum (Aubl.) Marchand & 15 & 0,46 & 1,27 & 0,42 & 2,15 & 0,88 \\
\hline Clusiaceae & Rheedia acuminata (Ruiz. \& Pav.) Planch. \& Triana & 8 & 0,24 & 1,27 & 0,15 & 1,66 & 0,39 \\
\hline Caesalpiniaceae & Macrolobium multijugum (DC.) Benth. & 5 & 0,15 & 1,27 & 0,19 & 1,62 & 0,34 \\
\hline Caesalpiniaceae & Crudia amazonica Spruce ex Benth. & 5 & 0,15 & 1,27 & 0,19 & 1,62 & 0,34 \\
\hline Ebenaceae & Diospyros guianensis (Aubl.) Gurke & 6 & 0,18 & 1,27 & 0,16 & 1,61 & 0,34 \\
\hline Bombacaceae & Quararibea guianensis Aubl. & 5 & 0,15 & 1,27 & 0,10 & 1,52 & 0,25 \\
\hline Combretaceae & Buchenavia oxycarpa (Mart.) Eichler & 3 & 0,09 & 1,27 & 0,13 & 1,50 & 0,22 \\
\hline Chrysobalanaceae & Licania Iongistyla (Hook. f.) Fritsch & 5 & 0,15 & 1,27 & 0,07 & 1,50 & 0,18 \\
\hline Euphorbiaceae & Drypetes variabilis Uittien & 4 & 0,12 & 1,27 & 0,06 & 1,46 & 0,18 \\
\hline Chrysobalanaceae & Couepia paraensis (Mart. \& Zucc.) Benth. ex Hook. f. & 2 & 0,06 & 1,27 & 0,12 & 1,45 & 0,16 \\
\hline Caesalpiniaceae & Poecilanthe effusa (Huber) Ducke & 3 & 0,09 & 1,27 & 0,07 & 1,43 & 0,09 \\
\hline Chrysobalanaceae & Hirtella racemosa Lam. & 2 & 0,06 & 1,27 & 0,03 & 1,37 & 0,09 \\
\hline Melastomataceae & Mouriri grandiflora DC. & 2 & 0,06 & 1,27 & 0,03 & 1,36 & 0,60 \\
\hline Caesalpiniaceae & Macrolobium pendulum Willd. ex Vogel & 13 & 0,40 & 0,64 & 0,20 & 1,23 & 0,56 \\
\hline
\end{tabular}




\begin{tabular}{|c|c|c|c|c|c|c|c|}
\hline Famílias & Espécies & $\mathrm{Ni}$ & DR & FR & DOMR & IVI & IVC \\
\hline Rubiaceae & Genipa americana L. & 2 & 0,06 & 0,64 & 0,10 & 0,80 & 0,13 \\
\hline Sapotaceae & Pouteria paraensis (Standl.) Baehni. & 3 & 0,09 & 0,64 & 0,04 & 0,77 & 0,11 \\
\hline Lecythidaceae & Allantoma lineata (Mart. \& 0. Berg) Miers & 2 & 0,06 & 0,64 & 0,05 & 0,75 & 0,11 \\
\hline Moraceae & Ficus maxima Mill. & 1 & 0,03 & 0,64 & 0,08 & 0,75 & 0,11 \\
\hline Icacinaceae & Poraqueiba guianensis Aubl. & 1 & 0,03 & 0,64 & 0,08 & 0,75 & 0,10 \\
\hline Mimosaceae & Enterolobium timbouva Mart. & 1 & 0,03 & 0,64 & 0,07 & 0,74 & 0,10 \\
\hline Mimosaceae & Inga alba (Sw.) Willd. & 2 & 0,06 & 0,64 & 0,04 & 0,73 & 0,10 \\
\hline Apocynaceae & Parahancornia amapa (Huber) Ducke & 2 & 0,06 & 0,64 & 0,03 & 0,73 & 0,08 \\
\hline Burseraceae & Protium giganteum Engl. & 1 & 0,03 & 0,64 & 0,05 & 0,72 & 0,07 \\
\hline Mimosaceae & Abarema jupunba (Willd.) Britton \& Killip & 1 & 0,03 & 0,64 & 0,04 & 0,71 & 0,06 \\
\hline Chrysobalanaceae & Licania heteromorpha Benth. & 1 & 0,03 & 0,64 & 0,03 & 0,70 & 0,06 \\
\hline Sapotaceae & Manilkara siqueiraei Ducke & 1 & 0,03 & 0,64 & 0,03 & 0,69 & 0,05 \\
\hline Aquifoliaceae & Ilex inundata Poepp. ex Reissek & 1 & 0,03 & 0,64 & 0,02 & 0,69 & 0,05 \\
\hline Lauraceae & Nectandra myriantha Meisn. & 1 & 0,03 & 0,64 & 0,02 & 0,69 & 0,05 \\
\hline Myrtaceae & Eugenia diplocampta Diels & 1 & 0,03 & 0,64 & 0,02 & 0,69 & 0,05 \\
\hline Bombacaceae & Matisia paraensis Huber. & 1 & 0,03 & 0,64 & 0,02 & 0,68 & 0,05 \\
\hline Mimosaceae & Zygia latifolia (L.) Fawc \& Rendle & 1 & 0,03 & 0,64 & 0,02 & 0,68 & 0,05 \\
\hline Papilionaceae & Platymiscium filipes Benth. & 1 & 0,03 & 0,64 & 0,01 & 0,68 & 0,04 \\
\hline Euphorbiaceae & Amanoa guianensis Aubl. & 1 & 0,03 & 0,64 & 0,01 & 0,68 & 0,04 \\
\hline Rhyzophoraceae & Cassipourea guianensis Aubl. & 1 & 0,03 & 0,64 & 0,01 & 0,68 & 0,04 \\
\hline
\end{tabular}

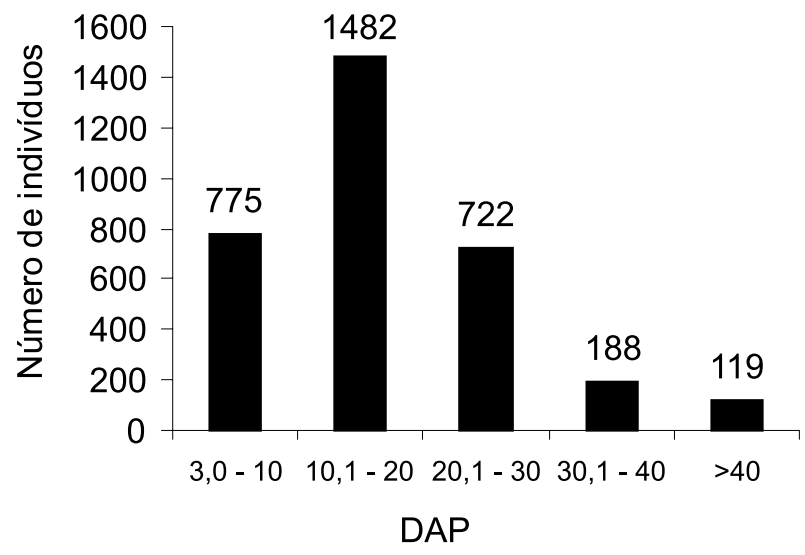

Figura 4 - Agrupamento do número dos indivíduos em classes diamétricas em 4 ha de floresta de várzea, em Santa Bárbara do Pará, estado do Pará. Brasil.

Na estratificação dos indivíduos em classes de altura (Fig. 5), observa-se a forma piramidal "J" invertido com a maior concentração de representantes nas classes II (819 indivíduos) e intermediária III. Nesta última, a mais representativa foi E. oleracea, contribuído com 22,65\%, correspondendo a 241 indivíduos do total.

A similaridade obtida pelo índice de Sorenson (IS) foi de 0,6 (Tabela 3). Esse valor acima de 0,5 indica alta semelhança entre as parcelas. No geral, a diversidade obtida pelo índice de Shannon

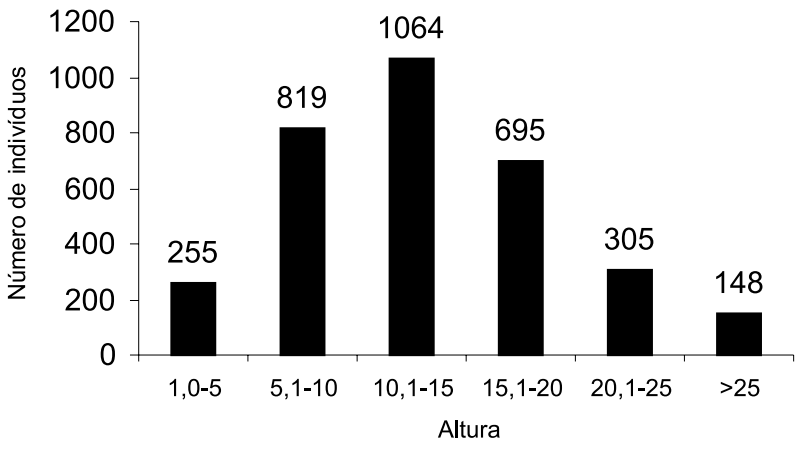

Figura 5 - Relação dos indivíduos distribuídos em classes de altura em 4 ha de floresta de várzea, em Santa Bárbara do Pará, estado do Pará, Brasil.

(H') foi de 2,69 com uma equabilidade (E') de 0,63, (Tab. 3). Para melhor explicar a similaridade existente na área estudada, apresenta-se a Tab. 4 composta pela matriz de similaridade, baseada no índice de Sorenson (IS $=2 \mathrm{c} /(\mathrm{a}+\mathrm{b}))$. A maior similaridade ocorreu entre as parcelas 3 e $2(0,70)$ e a menor entre as parcelas 4 e $2(0,51)$. É importante ressaltar que as parcelas de maior similaridade são aquelas que estão mais distantes do rio principal, porém, mais próximas entre si, e as de menor similaridade, as mais distantes entre si. Desse modo, em auxílio ao entendimento do índice de diversidade de Shannon-Weaver $\left(\mathrm{H}^{\prime}\right)$, a curva espécie-área (Fig. 6) mostra que 2,5 ha são suficientes para expressar a diversidade vegetal desse ecossistema. Desta 
Tabela 3 - Número de espécies (Nsp), Índice de diversidade de ShannonWeaver ( $\mathrm{H}^{\prime}$ ), Índice de equabilidade (E'), e Índice de similaridade de Sorenson (IS') para quatro hectares de floresta de várzea em Santa Bárbara do Pará, estado do Pará, Brasil.

\begin{tabular}{ccccc}
\hline Parcelas & Nsp & H'$^{\prime}$ & E'$^{\prime}$ & IS' $^{\prime}$ \\
\hline 1 & 33 & 2,237 & 0,634 & \\
2 & 39 & 2,709 & 0,739 & 0,6 \\
3 & 41 & 2,631 & 0,708 & \\
4 & 42 & 2,501 & 0,669 & \\
& 70 & 2,694 & 0,632 & 0,6 \\
\hline
\end{tabular}

Tabela 4 - Matriz de similaridade em 4 áreas por intermédio do índice de Sorenson (IS) com 70 espécies encontradas em quatro hectares de floresta de várzea em Santa Bárbara do Pará, estado do Pará, Brasil.

\begin{tabular}{ccccc}
\hline Parcelas & Parcela 1 & Parcela 2 & Parcela 3 & Parcela 4 \\
\hline 1 & -- & & & \\
2 & 0,6027 & -- & & \\
3 & 0,6400 & 0,7000 & - - & \\
4 & 0,6053 & 0,5185 & 0,5301 & - - \\
\hline
\end{tabular}

forma, a curva espécie-área confirma a não expressividade do índice de diversidade (2.69) para a área estudada.

\section{DISCUSSÃO}

A relação do número de famílias, gêneros e espécies deste estudo são similares a outros trabalhos realizados em ecossistema de várzea na Amazônia brasileira, como os de Conceição (1990), Rabelo et al. (2002) e Almeida et al. (2002). O baixo número de famílias e espécies nesse ecossistema pode estar condicionado ao próprio ambiente, ou seja, solos com alto nível de saturação, onde apenas espécies adaptadas conseguem sobreviver e disseminar de modo a dominar a paisagem. Isto foi comprovado por Junk (1980) relacionando a alta sedimentação em solos inundáveis como limitante para certos grupos de espécies arbóreas, favorecendo outras e por Scarano et al. (1994) que afirmam que a sobrevivência vegetal em áreas inundáveis não depende apenas de um único mecanismo adaptativo. Um exemplo da predominância de determinados grupos são as leguminosas (Caesalpiniaceae, Mimosaceae e Papilionaceae). Esse grupo utiliza a associação com a bactéria Rhizobium como estratégia em solos mal drenados, para compensar o déficit de nitrogênio, o que ajuda no estabelecimento da espécie (Moreira \& Silva, 1993). Porém, isso não deve ser entendido como regra geral, pois, segundo Sampaio et al. (2000) evidenciaram as leguminosas com número reduzido de espécies em áreas alagadas do Distrito Federal e, segundo esses autores, essas variaçốes podem estar relacionadas

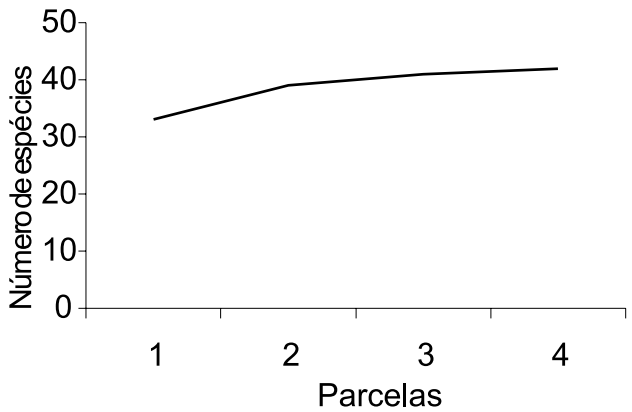

Figura 6 - Número de espécies em função do número de unidades amostrais em quatro hectares de floresta de várzea, em Santa Bárbara do Pará, estado do Pará. Brasil.

com fatores do meio como topografia, grau de encharcamento do solo e luminosidade.

Arecaceae, Papilionaceae e Caesalpiniaceae, em ecossistema de várzea, geralmente são mais abundantes. No caso de Arecaceae, a alta densidade pode estar relacionada à estratégia reprodutiva das espécies representantes como $E$. oleracea, que obteve maior densidade, provavelmente devido a sua capacidade reprodutiva por perfilhamento e germinação de sementes, como mostraram estudos de Pollak et al. (1995), Nogueira (1997) e Jardim (2000). Para Conceição (1990) e Almeida et al. (2002), a presença de Papilionaceae e Arecaceae, na maioria dos parâmetros fitossociológicos é decorrente das condiçōes favoráveis do meio ambiente e para Jardim (2000) por ter o maior número de espécies e indivíduos em áreas estuarinas. Para visualização comparativa das famílias e espécies citadas em floresta de várzea, elaborou-se a Tab. 5.

Neste estudo, a família mais importante foi Papilionaceae, representada por $P$. oficinalis. De acordo com Ducke \& Black (1958), as leguminosas são importantes na fisionomia da floresta amazônica. Em estudos realizadod por Bonadie \& Bacon (1999) em áreas alagadas de Trinidad, foi observado que a alta ocorrência de P. officinalis provavelmente seja para competir por dominância. Para Jardim (2000), em áreas de várzea, as palmeiras são consideradas dominantes em relação às outras espécies por estarem adaptadas às condiçōes de solos férteis e diretamente relacionadas com a água. Portanto, é comum encontrar no estuário amazônico, ampla dominância e elevados índices de valor de importância para Papilionaceae e Arecaceae. É notório que certos grupos possuem estratégias para estabelecimento em seu habitat. Assim, conseguem obter maior representatividade, volume, tamanho etc., é o caso de Papilionaceae com área basal de $39,44 \mathrm{~m}^{2} / \mathrm{ha}$ excedendo mais que o dobro a segunda (Caesalpiniaceae). Essa abrangência de superfície deve-se, principalmente, ao fato de Papilionaceae apresentar alta densidade e muitos indivíduos em classes diamétricas superiores, como $P$. officinalis observada como a espécie de maior área basal nesse estudo, situação que, logicamente, associa-se ao elevado número de indivíduos com 
Tabela 5 - Relação das famílias e espécies mais importantes em áreas de várzeas e seus respectivos índices fitossociológicos.

\begin{tabular}{|c|c|c|c|c|c|c|c|c|}
\hline Famílias & Dr & $\mathrm{Fr}$ & Dor & IVI & Área ha & Nível de amostragem & Local & Autor \\
\hline Arecaceae & 70,65 & -- & 45,85 & 54,70 & 5 & $\mathrm{DAP} \geq 10 \mathrm{~cm}$ & Combu, Belém - PA & Jardim(2000) \\
\hline Arecaceae & 72,06 & 4,50 & 23,73 & 100,29 & 5 & $\mathrm{DAP} \geq 5 \mathrm{~cm}$ & Lontra da Pedreira - AP & Rabelo et al. (2002) \\
\hline Arecaceae & 47,35 & 4,03 & 11,93 & 63,31 & & 11,93 & MazagãoAP & \\
\hline Papilionaceae & 25,84 & 4,88 & 37,50 & 68,21 & 4 & $\mathrm{DAP} \geq 10 \mathrm{~cm}$ & Sta Bárbara - PA & Este trabalho \\
\hline Arecaceae & 27,75 & 4,88 & 6,26 & 38,84 & & $\mathrm{CAP} \geq 10 \mathrm{~cm}$ & & \\
\hline Espécies & Dr & $\mathrm{Fr}$ & Dor & IVI & Área ha & Nível deamostragem & Local & Autor \\
\hline Euterpe oleracea & 56,35 & 24,55 & 32,46 & 37,79 & 5 & $\mathrm{DAP} \geq 10 \mathrm{~cm}$ & Combu, Belém-PA & Jardim(2000) \\
\hline Euterpe oleracea & 48,10 & 2,75 & 10,14 & 60,98 & 5 & $\mathrm{DAP} \geq 5 \mathrm{~cm}$ & Lontra da Pedreira - AP & Rabelo et al. (2002) \\
\hline Euterpe oleracea & 39,01 & 1,95 & 7,06 & 48,01 & 5 & $\mathrm{DAP} \geq 5 \mathrm{~cm}$ & Mazagão - AP & \\
\hline Pterocarpus officinalis & 14,00 & 5,98 & 15,81 & 11,93 & 1 & $\mathrm{DAP} \geq 10 \mathrm{~cm}$ & Sta Bárbara - PA & Almeidaet al.(2003) \\
\hline Pterocarpus officinalis & 21,36 & 2,55 & 32,66 & 56,57 & 4 & $\mathrm{DAP} \geq 10 \mathrm{~cm}$ & Sta Bárbara - PA & Este Trabalho \\
\hline Euterpe oleracea & 26,08 & 2,55 & 4,00 & 32,62 & & $\mathrm{CAP} \geq 10 \mathrm{~cm}$ & & \\
\hline
\end{tabular}

diâmetro expressivo. Bonadie \& Bacon (1999), em Trinidad, relataram que o desenvolvimento de P. officinalis possui estreitas relações com áreas alagadas. Desta forma, supõe-se que a elevada área basal de uma espécie pode estar intimamente ligada ao ajuste biológico da espécie com o regime de inundação.

Comparando-se as áreas basais de Papilionaceae e Arecaceae, e suas principais espécies, P. officinalis e E. oleracea; Papilionaceae merece destaque, fato que pode inferir-se a característica de desenvolvimento da própria espécie em possuir crescimento diamétrico avantajado, enquanto a outra, apresenta desenvolvimento diamétrico limitado. Sendo assim, todos os indivíduos contribuem para expressar a área basal por hectare, onde, nesse estudo, resultou num valor de $26,29 \mathrm{~m}^{2} \cdot \mathrm{ha}^{-1}$, sendo que nesse caso, vários fatores possivelmente estejam atuando conjuntamente como: aporte de nutrientes, fluxo de maré e nível de antropização. Ramos (2000) e Bentes-Gama et al. (2002), encontraram valores de área basal por hectare similar a esse estudo, em torno de $26,0 \mathrm{~m}^{2} \cdot \mathrm{ha}^{-1}$ e $23,4 \mathrm{~m}^{2} \cdot \mathrm{ha}^{-1}$, respectivamente. Porém, Santos (2002), nas várzeas do rio Juba, Cametá - PA, encontrou $40 \mathrm{~m}^{2} \cdot \mathrm{ha}^{-1}$, o que está bem acima dos demais trabalhos, fato que evidencia a diferença entre áreas manejadas e áreas não manejadas.

A estrutura diamétrica configurou-se em uma curva exponencial negativa, evidenciando alta concentração de indivíduos de E. oleracea nas classes iniciais. A curva exponencial negativa constitui uma das características de florestas tropicais, ou seja, muitos indivíduos no sub-bosque florestal onde representantes das classes superiores (V e VI) contribuem de modo satisfatório para a manutenção do número de indivíduos. Para Jardim (1995), a alta ocorrência de indivíduos nas classes iniciais é característica de florestas tropicais nativas. Da mesma forma, a estratificação dos indivíduos em classes de altura configurou-se em uma curva exponencial negativa, com muitos indivíduos jovens nas classes iniciais à intermediária, indicando ser uma floresta de extrato clímax. Santos (2002), na várzea do rio Juba, Cametá - PA, mostrou que cerca de $72 \%$ dos indivíduos enquadram-se nas classes iniciais de CAP e altura e que determinadas espécies como E. oleracea, são responsáveis pela distribuição "J" invertido. Esse tipo de distribuição para as classes de tamanho pode ser observado em diversos trabalhos em floresta tropical (Jardim 2000; Rabelo et al. 2002; Gama et al. 2002).

Em florestas tropicais, os índices de similaridade e diversidade são inversamente proporcionais. Nas várzeas é comum haver alta similaridade e, conseqüentemente, baixa diversidade, fato que pode associar-se a fatores do meio. Neste estudo a similaridade obtida de 0,6 pode ser compreendida como alta similaridade. Desta forma, observou-se maior similaridade entre as parcelas $2 \mathrm{e}$ 3 que estão mais próximas entre si, que são de topografia mais baixa, apresentam maior número de furos e intenso fluxo de marés. A similaridade parece ter relação direta com os fatores do meio, como fluxo de maré e permanência de inundação, bem como com a distância que possivelmente influencia no fluxo gênico. Almeida et al. (2002), no estuário amazônico, relataram, entre outros fatores, que a variação no ambiente, efeitos de zonação, altura de inundação e velocidade da água, influenciam diretamente na similaridade entre áreas.

Provavelmente, a diversidade é efeito direto da variação do ambiente, onde, nesse estudo, o índice foi considerado baixo $(2,69)$, porém, compatível com as áreas de várzea na Amazônia conforme mostraram Pires (1973) e Ayres (1993) que encontraram valores similares. De acordo com Pires \& Koury (1959), a baixa diversidade florística pode estar relacionada com as limitaçôes do ambiente. Almeida et al. (2003), analisando 25 plots de $400 \mathrm{~m}^{2}$ em floresta de várzea, em Santa Bárbara do Pará, encontraram índice de diversidade (Shannon) em torno de 3,15 e salientaram que a diversidade é baixa devido ao efeito da maré e à baixa estabilidade dos solos hidromórficos recentes. Para Miranda \& Diógenes (1998), o índice de diversidade para 
florestas tropicais varia entre 1,5 e 3,5 e raramente ultrapassa a 4,5. A diversidade é comprovada pelo índice de equabilidade que é diretamente proporcional e que por sua vez correlaciona a diversidade e o maior valor que esta pode alcançar. Segundo Magurran (1988), a equabilidade é compreendida entre 0 e 1. Quando esta se aproxima de 1, significa que há alta diversidade e que as espécies são teoricamente abundantes entre as parcelas. Nesta pequisa, a equabilidade de 0,63 demonstra que as espécies não são igualmente abundantes. Estudos de Martorano (2000), em várzea no município de Belém (PA) e Jardim et al (2004) nas florestas de várzeas dos municípios de Breves e Melgaço (PA), mostraram valores de equabilidade das espécies próximos ao encontrado nesse estudo.

\section{AGRADECIMENTOS}

Ao Conselho Nacional de Desenvolvimento Científico e Tecnológico/Programa Norte de Pós-Graduação em apoiar todas atividades de campo pelo projeto "Dinâmica de fragmentação das florestas fluviais no estuário amazônico e alternativas de mitigação dos efeitos pelo manejo florestal do açaizeiro (Euterpe oleracea Mart.)", Processo 550155/01-5.

\section{BIBLIOGRAFIA CITADA}

Ayres, J.M. 1993. As matas de várzea do Mamirauá: Médio rio Solimões. Brasília, DF. Sociedade Civil Mamirauá. v.1. 123pp.

Almeida, S.S.; Amaral, D.D. Silva, A.S.L. 2002. Estrutura e fitossociologia de florestas de várzea no estuário amazônico. ECOLAB. CD ROOM.

Almeida, S.S.; Amaral, D.D. Silva, A.S.L. 2003. Inventário floristico e análise fitossociológica dos ambientes do parque de Gumna, município de Santa Bárbara, Pará. Relatório Técnico Final. p.110-112.

Bentes-Gama, M.M.; Scolforo, J.R.S. Gama, J.R.V. 2002. Potencial produtivo de madeira e palmito de uma floresta secundária de várzea baixa no estuário amazônico. Revista Árvore, 26(3): 311319.

Bonaide, W.A.; Bacon, P.R. 1999. The structure and ecology of Mauritia setigera palm swamp Forest in native swamp, Trinidad. Tropical Ecology, 40(2): 199-206.

Conceição, M.C.A. 1990. Análise estrutural de uma floresta de várzea no Estado do Pará. Curitiba: Dissertação de mestrado. Universidade Federal do Paraná, Curitiba, Paraná. 107 pp.

Cronquist, A. 1981. A integrated system of classification of flowering plants. New York, Columbia University Press. p. 592 - 605.

Ducke, A.; Black, G.A. 1958. Notas sobre a fitogeografia da Amazônia brasileira. Instituto de Pesquisa Agronômico do Norte, 29: 3-62.

Gama, J.R.V.; Botelho, S.A. Bentes-Gama, M.M. 2002. Composição florística e estrutura da regeneração natural de floresta secundária de várzea baixa no estuário amazônico. Revista Arvore, 26(1): 559-566.

Jardim, F.C.S. 1995. Comportamento da regeneraçāo natural de espécies arbóreas em diferentes intensidades de desbastes por anelamento, na região de Manaus - AM. Tese de doutorado. Universidade Federal de Viçosa, Minas Gerais. 162 pp.
Jardim, M.A.G.; Cunha A.C.C. 1998. Caracterização de populações nativas de palmeiras do estuário amazônico. Boletim Museu Paraense Emílio Goeldi. ser. Botânica, 14(1): 33-41.

Jardim, M.A.G. 2000. Morfologia e ecologia do açaizeiro Euterpe oleracea Mart. e das etnovariedades espada e branco em ambiente de várzea do estuário amazônico. Tese de doutorado. Universidade Federal do Pará/Museu Paraense Emílio Goeldi. Belém, Pará. 119 pp.

Jardim, M.A.G.; Amaral, D.D.; Santos, G.C.; Medeiros, T.D.S.; Silva, C.A.; Francez, D.C.; Neto, S.V.C. 2004. Análise florística e estrutural para avaliação da fragmentação nas florestas de várzea do estuário amazônico. In: Jardim, M.A.G.; Mourão, L.; Grossmann, M. (Org.) - Açaí - possibilidades e limites para o desenvolvimento sustentável no estuário amazônico. Belém: MPEG, pg.101-121.

Junk, W. J. 1980. Áreas inundáveis. Um desafio para a limnologia. Acta Amazonica, 10(4): 775-796.

Legendre, L.; Legendre, P. 1983. Numerical ecology. Elsevier. New York. 419 pp.

Lima, R.R.; Tourinho, M.M.; Costa, J.P.C. 2000. Várzeas fluviomarinhas da Amazônia brasileira: Características e possibilidades agropecuárias. Belém. Faculdade de Ciências do Pará.342 pp.

Macedo, D.S.; Anderson,A.B. 1993. Early ecological changes associated with logging in a Amazon floodplain. Biotropica 25(2): 151-163.

Magurran, A.E. 1988. Ecological diversity and its measurement. Princenton Univ. Press. New Jersey. 179 pp.

Martorano, P.G. 2000. Avaliação estrutural de uma floresta de várzea localizada na área de influência da macrodrenagem, bacia do Una, na regiāo urbana de Belém, Pará. Dissertação de mestrado. Faculdade de Ciências Agrárias do Pará, Belém, Pará. 81 pp.

Miranda, I.S.; Diógenes, M.B. 1998. Caracterização florística, fisionômica e estrutural da vegetação da floresta nacional do Macauã. Rio Branco-AC. Relatório técnico. 118 pp.

Moreira, F.M.S.; Silva, M.F. 1993. Associação rizóbio-leguminosas na Amazônia. Boletim do Museu Paraense Emílio Goeldi, sér. Botânica 9(2): 129-141.

Nogueira, O.L. 1997. Regeneração, manejo e exploração de açaizais nativos de várzea do estuário amazônico. Tese de doutorado. Universidade Federal do Pará/Museu Paraense Emílio goeldi/ Empresa Brasileira de Pesquisa Agropecuária. Belém. 149 pp.

Pielou, E.C. 1977. Mathematical ecology. Wiley, New York. 165 p.

Pires, J. M.; Koury, H. M. 1959. Estudo de um trecho de mata de várzea próximo a Belém. Boletim Técnico Instituto de Pesquisa Agronômico do Nordeste 36: 3 - 44.

Pires, J. M. 1973. Tipos de vegetação da Amazônia. Boletim do Museu Paraense Emílio Goeldi, série Botânica 20(1): 179-202.

Pollak, H.; Mattos, M.; Uhl, C. 1995. A profile of palm heart extraction in the Amazon estuary. Human Ecology 23(3): 357384.

Rabelo, F.G. 1999. Composição florística, estrutura e regeneração de ecossistemas florestais na região estuarina do Rio Amazonas-AmapáBrasil. Dissertação de Mestrado. Faculdade de Ciências Agrárias do Pará. Belém. 72 pp. 


\section{ACTA
AMAZONICA}

Rabelo, F.G.; Zarin, D.J.; Oliveira, F.A.; Jardim, F.C.S. 2002. Diversidade, composição florística e distribuição diamétrica do povoamento com DAP e" $5 \mathrm{~cm}$ em região do estuário do Amapá. Revista de Ciências Agrárias, 37: 91-112.

Ramos, C.A.P. 2000. Possibilidades de otimização do uso florestal para pequenos produtores nas várzeas amazônicas: um estudo na costa amapaense. Dissertação de mestrado. Faculdade de Ciências agrárias do Pará, Belém, Pará. 112 p.

Sampaio, A.B.; Walter, B.M.T.; Felfili, J.M. 2000. Diversidade e distribuição de espécies arbóreas em duas matas de galeria na micro-bacia do Riacho Fundo, Distrito Federal. Acta Botanica Brasílica, 14(2): 197-214.

Sanchez, M.; Pedroni, F.; Leitão-Filho, H. de F.; Cezar, O. 1999. Composição florística de um trecho de floresta ripária na mata atlântica em Picinguaba, Ubatuba, São Paulo. Revista Brasileira Botanica, 22 (1): 31-42.
Santos, S.R.M. 2002. Estrutura e estimativa de biomassa dos sistemas agroflorestais das várzeas do rio Juba, Cametá, Pará. Dissertação de mestrado. Faculdade de Ciências Agrárias do Pará, Belém, Pará. 50 p.

Scarano, F.R.; Cattânio, J.H.; Crawford, R.M.M. 1994. Root carbohidrate storage in young samplings of an Amazonian tidal várzea forest before the onset of wet season. Acta Botanica Brasilica, 8(2):129- 139 .

SUDAM (Superintendência do Desenvolvimento da Amazônia). 1993. Municípios Paraenses: Santa Bárbara do Pará. Governo do Estado do Pará, Belém: Secretaria de Planejamento (SEPLAN). Novos Municípios, 33: 36 pp.

Recebido em 26/11/2004

Aceito em 28/11/2006 